\title{
基于甲磺酰基的磺酰胺-氨醇作为催化醛的不对称炔基化反应新配体
}

\author{
金 薇，黄永波，万伯顺 \\ 中国科学院大连化学物理研究所, 辽宁大连 116023
}

\begin{abstract}
摘要：以商业易得的 (-)-麻黄碱、(+)-伪麻黄碱和各种手性氨醇为主要原料,经过简单的两步反应,合成了一系列基于甲磺酰基 的磺酰胺-氨醇 (SAA) 配体, 并将其用于催化苯乙炔基锌对醛的不对称加成反应. 在没有 $\mathrm{Ti}\left(\mathrm{O}^{i} \mathrm{Pr}\right)_{4}$ 等金属试剂的条件下,配体表 现出较好的不对称诱导能力, 取得了高达 $83 \%$ ee.
\end{abstract}

关键词：磺酰胺-氨醇配体; 不对称加成; 炔基化反应; 醛

中图分类号: O643 文献标识码: A

\section{Methylsulfonyl-Based Sulfamide-Amine Alcohol as a Ligand for Enantioselective Alkynylation of Aldehydes}

\author{
JIN Wei, HUANG Yongbo, WAN Boshun* \\ Dalian Institute of Chemical Physics, Chinese Academy of Sciences, Dalian 116023, Liaoning, China
}

\begin{abstract}
Chiral methylsulfonyl-based sulfamide-amine alcohol (SAA) ligands were synthesized from commercially available starting materials in two simple steps. Methylsulfonyl-based SAA ligands catalyzed the asymmetric alkynylation of various aldehydes using alkynylzinc to provide chiral propargyl alcohols with moderate to good enantioselectivity up to $83 \%$ ee.
\end{abstract}

Key words: sulfamide-amine alcohol; enantioselective addition; alkynylation; aldehyde

The catalytic enantioselective addition of terminal alkynes to aldehydes is a very useful method for the synthesis of chiral propargyl alcohols, which are important versatile building blocks of many biologically active compounds and natural products [1-4]. In recent years, chiral ligands, such as $\mathrm{N}$-methyl ephedrine [5,6], sulfonamide alcohol [7,8], BINOL and its derivatives [9-12], have successfully been used in this reaction. However, $\mathrm{Ti}\left(\mathrm{O}-{ }^{i} \mathrm{Pr}\right)_{4}$ or other metal species are usually necessary in most of these catalytic systems [13-17]. On the other hand [18-32], efficient ligands that can be prepared from cheap and easily available starting materials in a few synthetic steps and that are designed for facile structural variations are still particularly useful. The design of chiral ligands is the key to new enantioselective catalysts from a practical viewpoint $[33,34]$. We have reported a series of chiral $p$-tolyl sulfonyl (Ts)-based sulfamide-amine alcohol (SAA) ligands for asymmetric diethylzinc addition to aldehydes without the use of a titanium complex [35]. We also found that Ts-based and trifluoro- methanesulfonyl (Tf)-based SAA were also effective for the asymmetric alkynylation of carbonyl compounds [36-38]. In order to understand the electronic and steric effects of chiral SAA on asymmetric alkynylation of aldehydes and as part of extending the application of our SAA ligands, we report methylsulfonyl (Ms)-based SAA catalyzed enantioselective alkynylation of aldehydes under very mild conditions without using moisture sensitive $\mathrm{Ti}\left(\mathrm{O}-{ }^{i} \mathrm{Pr}\right)_{4}$ and $\mathrm{Zn}(\mathrm{OTf})_{2}$.

\section{Experimental}

\subsection{Typical procedure for the preparation of Ms-based SAA (4a)}

Methanesulfonyl chloride $(1.7 \mathrm{ml}, 22 \mathrm{mmol})$ was added dropwise over $1.5 \mathrm{~h}$ to $(S)$-alaninol $(1.37 \mathrm{~g}, 10 \mathrm{mmol})$ and triethylamine $(2.8 \mathrm{ml}, 20 \mathrm{mmol})$ in dry $\mathrm{CH}_{2} \mathrm{Cl}_{2}(60 \mathrm{ml})$ under argon at $-20{ }^{\circ} \mathrm{C}$. Stirring was continued at this tempera-

Received date: 2 February 2010 .

*Corresponding author. Tel: +86-411-84379260; Fax: +86-411-84379223; E-mail: bswan@dicp.ac.cn

Foundation item: Supported by the National Basic Research Program of China (973 Program, 2010CB833300).

English edition available online at ScienceDirect (http://www.sciencedirect.com/science/journal/18722067). 
ture for an additional $30 \mathrm{~min}$, after which the flask was kept at $-30{ }^{\circ} \mathrm{C}$ overnight. The cold solution was then washed with $0.1 \mathrm{~mol} / \mathrm{L} \mathrm{HC1}(10 \mathrm{ml} \times 2)$ and saturated aqueous $\mathrm{NaHCO}_{3}(15 \mathrm{ml})$. The organic phase was dried $\left(\mathrm{MgSO}_{4}\right)$ and the solvent evaporated to leave the corresponding aziridine (6) as a white solid. Without purification, the aziridine and (-)-ephedrine were dissolved in dry acetonitrile $(60 \mathrm{ml})$ and the mixture was stirred under reflux for 2 d. The solvent was evaporated under reduced pressure. The residue was purified by column chromatography to give the pure ligand. When the $\mathrm{R}$ group was changed to benzyl or methyl group, the triethylamine was changed to 4-dimethylaminopyridine (DMAP) and $\mathrm{K}_{2} \mathrm{CO}_{3}$, respectively, and the solvent was changed to acetonitrile. The spectral data of Ms-based ligands are listed below.

N-[(R)-2-[[(1R,2S)-1-hydroxy-1-phenylpropan-2-yl] (methyl)amino]-1-phenylethyl]methanesulfonamide (4a): white solid, $41 \%$ yield. mp: $127-128{ }^{\circ} \mathrm{C} ;{ }^{1} \mathrm{H}$ NMR: $\delta 1.09$ (d, $J=6.4 \mathrm{~Hz}, 3 \mathrm{H}), 2.07$ (s, 3H), 2.409 (s, 3H), 2.48 (dd, $J=$ $12 \mathrm{~Hz}, 1 \mathrm{H}), 2.67$ (dd, $J=4.4 \mathrm{~Hz}, 1 \mathrm{H}), 2.84$ (dt, $J=6.4 \mathrm{~Hz}$, $1 \mathrm{H}), 4.38$ (dd, $J=4.4 \mathrm{~Hz}, 1 \mathrm{H}), 4.67$ (d, $J=6.4 \mathrm{~Hz}, 1 \mathrm{H})$, 7.26-7.42 (m, $10 \mathrm{H}) ;{ }^{13} \mathrm{C}$ NMR: $\delta$ 10.52, 35.42, 42.01, $55.46,62.00,65.86,75.69,126.31,127.55,127.91,128.32$, $128.86,128.92,139.98,143.49$.

N-[(R)-2-[[(1S,2S)-1-hydroxy-1-phenylpropan-2-yl] (methyl)amino]-1-phenylethyl]methanesulfonamide

(4b): white solid, $31 \%$ yield. mp: $162-163{ }^{\circ} \mathrm{C}$; ${ }^{1} \mathrm{H}$ NMR: $\delta$ $0.65(\mathrm{~d}, J=6.8 \mathrm{~Hz}, 3 \mathrm{H}), 2.36(\mathrm{~m}, 4 \mathrm{H}), 2.54(\mathrm{~d}, J=6.4 \mathrm{~Hz}$, $1 \mathrm{H}), 2.61(\mathrm{~s}, 3 \mathrm{H}), 2.73(\mathrm{dd}, J=9.6$ and $12.8 \mathrm{~Hz}, 1 \mathrm{H}), 4.24$ $(\mathrm{d}, J=9.6 \mathrm{~Hz}, 1 \mathrm{H}), 4.58(\mathrm{dd}, J=6.4$ and $9.2 \mathrm{~Hz}, 1 \mathrm{H})$, $7.22-7.41(\mathrm{~m}, 10 \mathrm{H}) ;{ }^{13} \mathrm{C}$ NMR: $\delta 8.40,37.29,42.10,56.10$, $58.71,66.07,74.96,127.14,127.52,127.15,128.49$, 129.23, 140.24, 141.93 .

\section{N-[(S)-2-[[(1R,2S)-1-hydroxy-1-phenylpropan-2-yl]} (methyl)amino]-1-phenylethyl]methanesulfonamide (4c): white solid, $25 \%$ yield. mp: $110-112{ }^{\circ} \mathrm{C} ;{ }^{1} \mathrm{H}$ NMR: $\delta 0.97$ (d, $J=6.8 \mathrm{~Hz}, 3 \mathrm{H}), 2.24$ (s, 2H), 2.37 (s, 3H), 2.49 (dd, $J=$ 4.8 and $12.8 \mathrm{~Hz}, 1 \mathrm{H}), 2.63(\mathrm{dd}, J=12.8$ and $10 \mathrm{~Hz}, 1 \mathrm{H})$, $2.88(\mathrm{~m}, J=6.8$ and $5.6,1 \mathrm{H}), 4.45(\mathrm{dd}, J=10$ and $4.8 \mathrm{~Hz}$, $1 \mathrm{H}), 4.64(\mathrm{~d}, J=5.6 \mathrm{~Hz}, 1 \mathrm{H}), 7.25-7.45(\mathrm{~m}, 10 \mathrm{H}) ;{ }^{13} \mathrm{C}$ NMR: $\delta 8.95,39.36,41.94,55.74,58.92,64.22,76.84$, $126.77,127.50,127.82,128.26,128.71,128.94,140.26$, 143.07 .

N-[(S)-2-[[(1S,2S)-1-hydroxy-1-phenylpropan-2-yl] (methyl)amino]-1-phenylethyl]methanesulfonamide (4d): white solid, $20 \%$ yield. mp: $149-151{ }^{\circ} \mathrm{C}$; ${ }^{1} \mathrm{H}$ NMR: $\delta$ $0.67(\mathrm{~d}, J=6.8 \mathrm{~Hz}, 3 \mathrm{H}), 2.42(\mathrm{dd}, J=12.8$ and $6.0 \mathrm{~Hz}, 1 \mathrm{H})$, 2.37 (s, 3H), 2.56-2.59 (m, 1H), 2.61 (s, 3H), 2.75 (dd, $J=$ 9.2 and $12.8 \mathrm{~Hz}, 1 \mathrm{H}), 4.25(\mathrm{~d}, J=9.6 \mathrm{~Hz}, 1 \mathrm{H}), 4.59(\mathrm{dd}, J=$ 6.0 and $9.2 \mathrm{~Hz}, 1 \mathrm{H}), 7.30-7.41(\mathrm{~m}, 10 \mathrm{H}) ;{ }^{13} \mathrm{C} \mathrm{NMR}: \delta 8.41$, 37.37, 42.11, 56.07, 58.66, 65.97, 75.00, 127.14, 127.51, $128.15,128.50,129.01,129.23,140.19,141.91$.
N-[(R)-1-[[(1R,2S)-1-hydroxy-1-phenylpropan-2-yl] (methyl)amino]propan-2-yl]methanesulfonamide (5): white solid, $41 \%$ yield. mp: $124-125{ }^{\circ} \mathrm{C} ;{ }^{1} \mathrm{H}$ NMR: $\delta 0.70$ (d, $J=4.8$ and $6.4 \mathrm{~Hz}, 3 \mathrm{H}), 1.22(\mathrm{dd}, J=12.8$ and $6.4 \mathrm{~Hz}$, $3 \mathrm{H}), 2.20-2.24(\mathrm{~m}, 1 \mathrm{H}), 2.33(\mathrm{~d}, J=5.2 \mathrm{~Hz}, 3 \mathrm{H}), 2.49$ (dd, $J$ $=8.8$ and $12.8 \mathrm{~Hz}, 1 \mathrm{H}), 2.59-2.65(\mathrm{~m}, 1 \mathrm{H}), 3.03(\mathrm{~d}, J=4$ $\mathrm{Hz}, 3 \mathrm{H}), 3.60(\mathrm{~d}, J=6 \mathrm{~Hz}, 1 \mathrm{H}), 4.26(\mathrm{dd}, J=4.4$ and 9.6 $\mathrm{Hz}, 1 \mathrm{H}), 7.24-7.35$ (m, 5H); ${ }^{13} \mathrm{C}$ NMR: $\delta$ 7.96, 20.41, $37.91,41.92,48.02$, 58.39, 65.93, 75.03, 127.51, 128.12, $128.51,141.84$.

N-[(R)-1-[[(1R,2S)-1-hydroxy-1-phenylpropan-2-yl] (methyl)amino]-3-phenylpropan-2-yl]methanesulfonami de (6): white solid, $48 \%$ yield. mp: $104-105{ }^{\circ} \mathrm{C}$; ${ }^{1} \mathrm{H}$ NMR: $\delta$ $0.74(\mathrm{~d}, J=6.8 \mathrm{~Hz}, 3 \mathrm{H}), 2.30(\mathrm{~s}, 3 \mathrm{H}), 2.36(\mathrm{~s}, 3 \mathrm{H})$, $2.56-2.68(\mathrm{~m}, 3 \mathrm{H}), 2.71(\mathrm{dd}, J=2.4$ and $6.8 \mathrm{~Hz}, 1 \mathrm{H}), 3.06$ $(\mathrm{dd}, J=4.4$ and $14 \mathrm{~Hz}, 1 \mathrm{H}), 3.71(\mathrm{~m}, 1 \mathrm{H}), 4.31(\mathrm{~d}, J=9.6$ $\mathrm{Hz}, 1 \mathrm{H}), 7.22-7.41(\mathrm{~m}, 10 \mathrm{H}) ;{ }^{13} \mathrm{C}$ NMR: $\delta 8.18,36.66$, 40.16, 41.11, 55.54, 59.91, 66.66, 75.22, 127.07, 127.64, $128.09,128.53,128.91,129.85,138.34,141.74$.

1.2 Typical procedure for asymmetric addition of phenylacetylene to aldehydes

Under argon, the chiral ligand $(10 \mathrm{~mol} \%, 0.025 \mathrm{mmol})$ was mixed with dry $n$-hexane $(1.0 \mathrm{ml})$ at room temperature and stirred for $10 \mathrm{~min}$. Then $\mathrm{Et}_{2} \mathrm{Zn}(10 \mathrm{wt} \%$ in $n$-hexane, 0.9 $\mathrm{ml})$ and phenylacetylene ( $54 \mu \mathrm{l}, 0.5 \mathrm{mmol})$ were added by a syringe. After the mixture was stirred at room temperature for another $1 \mathrm{~h}$, aldehyde $(0.25 \mathrm{mmol})$ was added. The resulting mixture was stirred for $24 \mathrm{~h}$ at room temperature. The reaction was quenched with aqueous $\mathrm{HCl}(5 \%)$ and the mixture was extracted with ether $(6 \mathrm{ml})$. The combined organic layer was washed with brine, dried over $\mathrm{Na}_{2} \mathrm{SO}_{4}$, and concentrated under vacuum. The residue was purified by flash column chromatography to give the product.

1,3-Diphenylprop-2-yn-1-ol (7a). Retention time, $t_{\text {major }}=$ $11.6 \mathrm{~min}$ and $t_{\text {minor }}=21.1 \mathrm{~min}$.

1-(2-Methylphenyl)-3-phenylprop-2-yn-1-ol (7b). Retention time, $t_{\text {major }}=9.3 \mathrm{~min}$ and $t_{\text {minor }}=21.1 \mathrm{~min}$. ${ }^{1} \mathrm{H} \mathrm{NMR}$ : $\delta 2.45(\mathrm{~s}, 3 \mathrm{H}), 2.58(\mathrm{~s}, 1 \mathrm{H}), 5.78(\mathrm{~s}, 1 \mathrm{H}), 7.17-7.29(\mathrm{~m}, 6 \mathrm{H})$, 7.42-7.45 (m, 2H), 7.69-7.70 (m, 1H); ${ }^{13} \mathrm{C}$ NMR: $\delta$ 19.1, $63.0,86.6,88.8,122.7,126.4,126.7,128.4,128.6,128.7$, $130.9,131.9,136.2,138.5$.

1-(3-methylphenyl)-3-phenylprop-2-yn-1-ol (7c) [14]. Retention time, $t_{\text {major }}=10.6 \mathrm{~min}$ and $t_{\text {minor }}=25.7 \mathrm{~min}$.

1-(3-Methoxyphenyl)-3-phenylprop-2-yn-1-ol (7d). Retention time, $t_{\text {major }}=16.8 \mathrm{~min}$ and $t_{\text {minor }}=29.6 \mathrm{~min} .{ }^{1} \mathrm{H}$ NMR: $\delta 2.52(\mathrm{~s}, 1 \mathrm{H}), 3.80(\mathrm{~s}, 3 \mathrm{H}), 5.64(\mathrm{~m}, 1 \mathrm{H}), 6.86-6.88$ (m, 1H), 7.16-7.19 (m, 2H), 7.29-7.31 (m, 4H), 7.44-7.47 $(\mathrm{m}, 2 \mathrm{H}) ;{ }^{13} \mathrm{C}$ NMR: $\delta$ 55.5, 65.1, 86.7, 88.9, 112.3, 114.3, $119.2,122.6,128.5,128.8,129.9,131.9,142.4,160.0$.

1-(4-Methoxyphenyl)-3-phenylprop-2-yn-1-ol (7e) 
Retention time, $t_{\text {major }}=15.1 \mathrm{~min}$ and $t_{\text {minor }}=30.4 \mathrm{~min} .{ }^{1} \mathrm{H}$ NMR: $\delta 3.39$ (s, 1H), $3.84(\mathrm{~s}, 3 \mathrm{H}), 5.96(\mathrm{~s}, 1 \mathrm{H}), 6.88-7.01$ (m, 2H), 7.28-7.32 (m, 4H), 7.46-7.49 (m, 2H), 7.65-7.68 $(\mathrm{m}, 1 \mathrm{H}) ;{ }^{13} \mathrm{C}$ NMR: $\delta 55.3,60.7,85.6,88.8,110.8,120.6$, $127.7,128.1,128.2,129.5,131.5,156.5$.

1-(3-Fluorophenyl)-3-phenylprop-2-yn-1-ol (7f). Retention time, $t_{\text {major }}=9.2 \mathrm{~min}$ and $t_{\text {minor }}=27.3 \mathrm{~min} .{ }^{1} \mathrm{H}$ NMR: $\delta 2.621(\mathrm{~s}, 1 \mathrm{H}), 5.67(\mathrm{~s}, 1 \mathrm{H}), 7.00-7.02(\mathrm{~m}, 1 \mathrm{H}), 7.29-7.36$ $(\mathrm{m}, 6 \mathrm{H}), 7.45-7.47(\mathrm{~m}, 2 \mathrm{H}) ;{ }^{13} \mathrm{C}$ NMR: $\delta$ 64.6, 87.1, 88.3, $113.8,114.0,116.4,115.6,128.6,129.0,130.3,132.0$, 143.0, 164.0.

1-(4-Fluorophenyl)-3-phenylprop-2-yn-1-ol (7g) [14]. Retention time, $t_{\text {major }}=9.0 \mathrm{~min}$ and $t_{\text {minor }}=26.2 \mathrm{~min}$.

1-(4-chlorophenyl)-3-phenylprop-2-yn-1-ol (7h). Retention time, $t_{\text {major }}=9.4 \mathrm{~min}$ and $t_{\text {minor }}=29.0 \mathrm{~min} .{ }^{1} \mathrm{H} \mathrm{NMR}$ : $\delta 2.78(\mathrm{~s}, 1 \mathrm{H}), 5.63(\mathrm{~s}, 1 \mathrm{H}), 7.27-7.35(\mathrm{~m}, 5 \mathrm{H}), 7.43-7.45$ (m, 2H), 7.50-7.52 (m, 2H); ${ }^{13} \mathrm{C}$ NMR: $\delta$ 64.5,87.1, 88.4, $122.3,128.3,128.5,128.9,131.1,131.9,134.3,139.2$.

1-(3,5-Dichlorophenyl)-3-phenylprop-2-yn-1-ol

(7i).

Retention time, $t_{\text {major }}=6.6 \mathrm{~min}$ and $t_{\text {minor }}=29.6 \mathrm{~min} .{ }^{1} \mathrm{H}$ NMR: $\delta 3.02(\mathrm{~s}, 1 \mathrm{H}), 5.59(\mathrm{~s}, 1 \mathrm{H}), 7.27-7.35(\mathrm{~m}, 4 \mathrm{H})$, 7.42-7.45 (m, 4H); ${ }^{13} \mathrm{C}$ NMR: $\delta$ 63.9, 87.5, 87.6, 121.9, 125.3, 128.0, 128.6, 129.1, 132.0, 135.3, 143.9.

1-(3-Trifluoromethylphenyl)-3-phenylprop-2-yn-1-ol (7j). Retention time, $t_{\text {major }}=7.6 \mathrm{~min}$ and $t_{\text {minor }}=36.7 \mathrm{~min} .{ }^{1} \mathrm{H}$ NMR: $\delta 2.70(\mathrm{~s}, 1 \mathrm{H}), 5.73(\mathrm{~s}, 1 \mathrm{H}), 7.23-7.35(\mathrm{~m}, 3 \mathrm{H})$, 7.45-7.51 (m, 3H), 7.58-7.60 (m, 1H), 7.77-7.79 (m, 1H), 7.87 (s, 1H); ${ }^{13} \mathrm{C}$ NMR: $\delta$ 64.6, 87.5, 88.1, 122.2, 123.7,125.4, 128.6, 129.1, 129.3, 130.3,131.0, 131.3, 132.0,
141.2

1-(4-Trifluoromethylphenyl)-3-phenylprop-2-yn-1-ol (7k). Retention time, $t_{\text {major }}=8.4 \mathrm{~min}$ and $t_{\text {minor }}=42.0 \mathrm{~min}$. ${ }^{1} \mathrm{H}$ NMR: $\delta 2.56(\mathrm{~s}, 1 \mathrm{H}), 5.74(\mathrm{~s}, 1 \mathrm{H}), 7.25-7.37(\mathrm{~m}, 3 \mathrm{H})$, 7.45-7.47 (m, 2H), 7.64-7.74 (m, 4H); ${ }^{13} \mathrm{C}$ NMR: $\delta$ 64.6, 87.5, 88.1, 122.2, 125.8, 127.2, 128.6, 129.1, 132.0, 144.6.

1-(1-Naphthyl)-3-phenylprop-2-yn-1-ol (7l) [14]. Retention time, $t_{\text {major }}=17.1 \mathrm{~min}$ and $t_{\text {minor }}=36.6 \mathrm{~min}$.

\section{Results and discussion}

As shown in Scheme 1, in the traditional method [39], the chiral amino alcohols (1) first reacted with two equivalent methanesulfonyl chloride to afford substituted derivatives (2). Treatment with $\mathrm{NaH}$ resulted in $\mathrm{N}$-methanesulfonyl aziridine (3). In this work, 1 was reacted with methanesulfonyl chloride to give $\mathbf{3}$ directly, and then without purification, reacted with natural chiral (-)-ephedrine or $(+)$-pseudoephedrine to give the corresponding Ms-based SAA ligands (4) in two simple steps. The Ms-based SAA ligands are cheap and very stable in air.

The enantioselective addition of alkynylzinc to benzaldehyde was first examined in the presence of 0.1 equivalent chiral Ms-based SAA ligand in toluene. As shown in Table 1 , the matching of the stereogenic centers and substituent on the ligands had a large effect on the reaction. 4a was more effective than $\mathbf{4 b}, \mathbf{4 c}$, and $\mathbf{4 d}$, which have the same substituent as 4a (Table 1, entries 1-4). Changing the substituent from phenyl to methyl or benzyl group led to less enan-
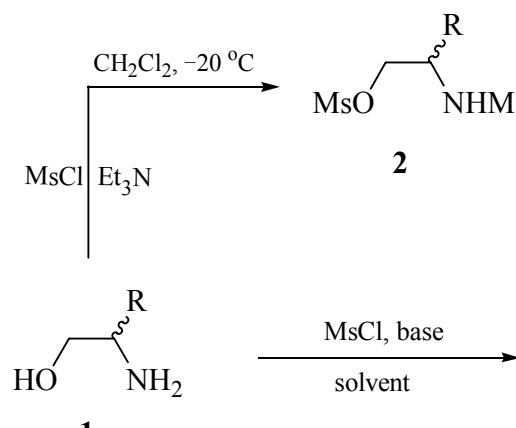

1

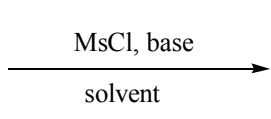

solven
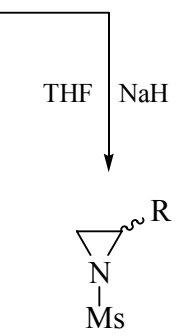

3
$\mathrm{R}=\mathrm{Ph}$, base: $\mathrm{Et}_{3} \mathrm{~N}$, solvent: $\mathrm{CH}_{2} \mathrm{Cl}_{2}$ $\mathrm{R}=\mathrm{Me}$, base: $\mathrm{K}_{2} \mathrm{CO}_{3}$, solvent: $\mathrm{CH}_{3} \mathrm{CN}$ $\mathrm{R}=\mathrm{Bn}$, base: DMAP, solvent: $\mathrm{CH}_{3} \mathrm{CN}$

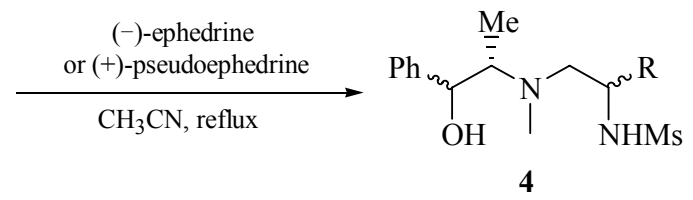<smiles>C[C@@H]([C@@H](O)c1ccccc1)N(C)C[C@H](NC([NH3+])=O)c1ccccc1</smiles>
$4 a$<smiles>CC(C(O)c1ccccc1)N(C)CC(NS(C)(=O)=O)c1ccccc1</smiles>
$4 d$<smiles>C[C@H](N(C)C[C@H](N)c1ccccc1)N(C)[C@H](O)c1ccccc1</smiles>

4b<smiles>CC(CN(C)C(C)[C@H](O)c1ccccc1)NS(C)(=O)=O</smiles><smiles>CC(C(O)c1ccccc1)N(C)CC(NS(C)(=O)=O)c1ccccc1</smiles><smiles>C[C@H](C(O)c1ccccc1)N(C)C[C@H](Cc1ccccc1)NS(C)(=O)=O</smiles>

$\begin{array}{cc} & \text { Yield } \\ \text { 4a } & 41 \% \\ \text { 4b } & 31 \% \\ \text { 4c } & 25 \% \\ \text { 4d } & 20 \% \\ \text { 5 } & 41 \% \\ \mathbf{6} & 48 \%\end{array}$

Scheme 1. Synthesis of Ms-based SAA. 
Table 1 Asymmetric alkynylation of benzaldehyde catalyzed by Ms-based SAA

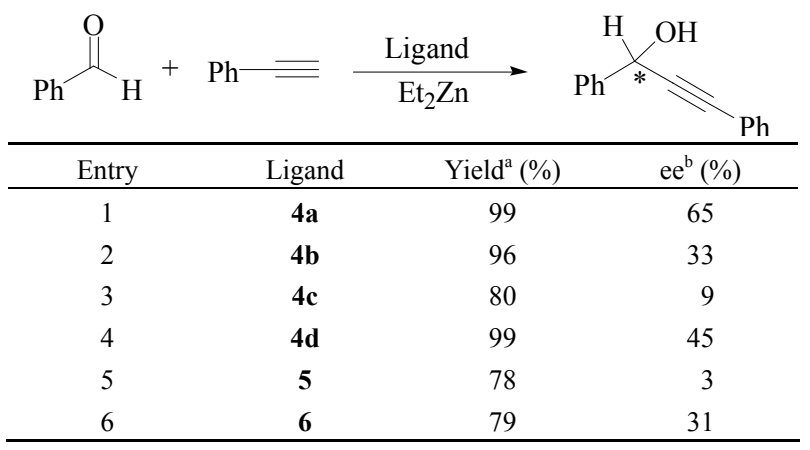

Reaction conditions: phenylacetylene: $\mathrm{Et}_{2} \mathrm{Zn}$ :aldehyde:ligand $=2.0: 2.2$ : 1:0.1, toluene $1 \mathrm{ml}, \mathrm{rt}, 24 \mathrm{~h}$.

${ }^{\mathrm{a}}$ Isolated yield.

${ }^{b}$ Determined by HPLC on a Chiracel OD-H column.

tioselectivity (Table 1, entries 5 and 6). Clearly, 4a was an effective chiral ligand for this asymmetric reaction.

To improve the enantioselectivity, the reaction conditions were optimized using benzaldehyde as the substrate (Table 2 ). The reactions were strongly influenced by the amount of ligand, reaction temperature, and solvent. At room temperature, increasing the amount of ligand $4 \mathbf{a}$ to $20 \mathrm{~mol} \% \mathrm{did}$ not improve the selectivity, while decreasing the amount of 4a to $5 \mathrm{~mol} \%$ gave a chiral product with only $43 \%$ ee (Table 2 , entries 1-3). Lower reaction temperatures did not improve the enantioselectivities (Table 2, entries 4 and 5). When the reaction was carried out in THF, low ee values were obtained (Table 2, entry 6). However, there was no enhancement in enantioselectivity when dichloromethane (DCM) and $\mathrm{Et}_{2} \mathrm{O}$ was used as the solvent (Table 2, entries 8 and 9). The best ee of $70 \%$ was obtained in $n$-hexane (Table 2, entry 7).

Having optimized the asymmetric alkynylation of benzaldehyde with phenylacetylene using $\mathbf{4 a}$, we decided to

Table 2 Optimization of reaction conditions

\begin{tabular}{|c|c|c|c|c|c|}
\hline Entry & $\begin{array}{c}\text { Content of } \mathbf{4 a} \\
(\mathrm{mol} \%)\end{array}$ & $t /{ }^{\circ} \mathrm{C}$ & Solvent & $\begin{array}{c}\text { Yield } \\
(\%)\end{array}$ & ee $(\%)$ \\
\hline 1 & 5 & $\mathrm{rt}$ & toluene & 66 & 43 \\
\hline 2 & 10 & $\mathrm{rt}$ & toluene & 99 & 65 \\
\hline 3 & 20 & $\mathrm{rt}$ & toluene & 98 & 65 \\
\hline 4 & 10 & 0 & toluene & 98 & 54 \\
\hline 5 & 10 & -20 & toluene & 98 & 31 \\
\hline 6 & 10 & $\mathrm{rt}$ & THF & 50 & 40 \\
\hline 7 & 10 & $\mathrm{rt}$ & $n$-hexane & 78 & 70 \\
\hline 8 & 10 & $\mathrm{rt}$ & DCM & 53 & 62 \\
\hline 9 & 10 & $\mathrm{rt}$ & $\mathrm{Et}_{2} \mathrm{O}$ & 99 & 62 \\
\hline
\end{tabular}

Reaction conditions are the same as in Table 1 except for the solvent and reaction temperature. screen various aldehydes. As can be seen from the summarized results in Table 3, 4a was efficient for all of the aromatic aldehydes studied. Propargylic alcohols were obtained with up to $83 \%$ ee and up to $91 \%$ yield.

Table 3 Asymmetric alkynylzinc addition to various aldehydes

\begin{tabular}{|c|c|c|c|}
\hline Entry & $\mathrm{R}$ & Yield (\%) & ee $(\%)$ \\
\hline 1 & $\mathrm{Ph}$ & 78 & 70 \\
\hline 2 & $o$-Me-Ph & 84 & 83 \\
\hline 3 & $m$-Me-Ph & 85 & 75 \\
\hline 4 & $m-\mathrm{MeO}-\mathrm{Ph}$ & 76 & 60 \\
\hline 5 & $p$-MeO-Ph & 65 & 60 \\
\hline 6 & $m-\mathrm{F}-\mathrm{Ph}$ & 70 & 66 \\
\hline 7 & $p$-F-Ph & 77 & 64 \\
\hline 8 & $p-\mathrm{Cl}-\mathrm{Ph}$ & 79 & 64 \\
\hline 9 & 3,5-2Cl-Ph & 75 & 68 \\
\hline 10 & $m-\mathrm{CF}_{3}-\mathrm{Ph}$ & 76 & 55 \\
\hline 11 & $p-\mathrm{CF}_{3}-\mathrm{Ph}$ & 66 & 60 \\
\hline 12 & 1-naphthyl & 91 & 81 \\
\hline
\end{tabular}

Reaction conditions are the same as in Table 1 except the solvent is $n$-hexane.

In order to explain the catalytic reaction mechanism with the Ms-based SAA ligands, proposed transition states are shown in Scheme 2. When 4a was used, the $\beta$-amino zinc atom acts as the Lewis acid center to active the aldehyde, while the oxygen atom of alkoxyl acts as the Lewis base center to independently activate the alkynylzinc nucleophile $[33,34]$. This explains how the SAA ligands catalyzed the enantioselective alkynylzinc addition to aldehydes without moisture sensitive $\mathrm{Ti}\left(\mathrm{O}-{ }^{i} \mathrm{Pr}\right)_{4}$ and $\mathrm{Zn}(\mathrm{OTf})_{2}$, which were needed in the literature [5-12,18-21].

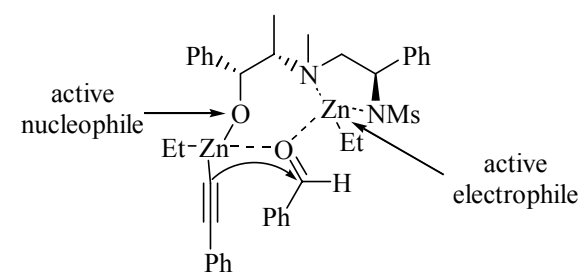

Scheme 2. Proposed transition states of the reaction.

\section{Conclusions}

New convenient chiral Ms-based SAA ligands were prepared from commercially available starting materials in two simple steps. Ms-based SAA 4a catalyzed the asymmetric alkynylation of aromatic aldehydes with up to $83 \%$ ee. The cheap Ms-based SAA 4a is a practical laboratory ligand for the enantioselective alkynylation of aromatic aldehydes under very mild conditions that do not need moisture sensitive $\mathrm{Ti}\left(\mathrm{O}-{ }^{i} \mathrm{Pr}\right)_{4}$ and $\mathrm{Zn}(\mathrm{OTf})_{2}$. 


\section{References}

1 Trost B M, Krische M J. J Am Chem Soc, 1999, 121: 6131

2 Fox M E, Li C, Marino J P Jr, Overman L E. J Am Chem Soc, 1999, 121: 5467

3 Corey E J, Cimprich K A. J Am Chem Soc, 1994, 116: 3151

4 Si Y G, Huang H, Jiang B. Chin J Org Chem, 2004, 11: 1389

5 Anand N K, Carreira E M. J Am Chem Soc, 2001, 123: 9687

6 Frantz D E, Fässler R, Carreira E M. J Am Chem Soc, 2000, 122: 1806

7 Xu Z Q, Wang R, Xu J K, Da C S, Yan W J, Chen C. Angew Chem, Int Ed, 2003, 42:5747

$8 \mathrm{Xu} \mathrm{Z} \mathrm{Q,} \mathrm{Chen} \mathrm{C,} \mathrm{Xu} \mathrm{J} \mathrm{K,} \mathrm{Miao} \mathrm{M} \mathrm{B,} \mathrm{Yan} \mathrm{W} \mathrm{J,} \mathrm{Wang} \mathrm{R.} \mathrm{Org}$ Lett, 2004, 6: 1193

9 Moore D, Pu L. Org Lett, 2002, 4: 1855

10 Gao G, Moore D, Xie R G, Pu L. Org Lett, 2002, 4: 4143

11 Xu M H, Pu L. Org Lett, 2002, 4: 4555

12 Li X S, Lu G, Kwok W H, Chan A S C. J Am Chem Soc, 2002, 124: 12636

13 Liu L, Wang R, Kang Y F, Chen C, Xu Z Q, Zhou Y F, Ni M, Cai H Q, Gong M Z. J Org Chem, 2005, 70: 1084

14 Kang Y F, Liu L, Wang R, Zhou Y F, Yan W J. Adv Synth Catal, 2005, 347: 243

15 Ni M, Wang R, Han Z J, Mao B, Da C, Liu L, Chen C. $A d v$ Synth Catal, 2005, 347: 1659

16 Chen C, Hong L, Xu Z Q, Liu L, Wang R. Org Lett, 2006, 8: 2277

17 Ding J, Shen Z X, Luo X Q, Chen W Y, Zhang Y W. Chin J Chem, 2006, 24: 1285

18 Pu L. Tetrahedron, 2003, 59: 9873

19 Cozzi P G, Hilgraf R, Zimmermann N. Eur J Org Chem, 2004: 4095

20 Lu G, Li Y M, Li X S, Chan A S C. Coord Chem Rev, 2005, 249: 1736
21 Trost B M, Weiss A H. Adv Synth Catal, 2009, 351: 963

22 Gao G, Xie R G, Pu L. Proc Nat Acad Sci, 2004, 101: 5417

23 Takita R, Yakura K, Ohshima T, Shibasaki M. J Am Chem Soc, 2005, 127: 13760

24 Gao G, Wang Q, Yu X Q, Xie R G, Pu L. Angew Chem, In Ed, 2006, 45: 122

25 Trost B M, Weiss A H, von Wangelin A J. J Am Chem Soc, 2006, 128: 8

26 Emmerson D P G, Hems W P, Davis B G. Org Lett, 2006, 8: 207

27 Xu Z Q, Lin L, Xu J K, Yan W J, Wang R. Adv Synth Catal, 2006, 348: 506

28 Wolf C, Liu S L. J Am Chem Soc, 2006, 128: 10996

29 Li Y M, Tang Y Q, Hui X P, Huang L N, Xu P F. Tetrahedron, 2009, 65: 3611

30 Zhong J C, Hou S C, Bian Q H, Yin M M, Na R S, Zheng B, Li Z Y, Liu S Z, Wang M. Chem Eur J, 2009, 15: 3069

31 Xu Z, Wu N, Ding Z H, Wang T, Mao J C, Zhang Y W. Tetrahedron Lett, 2009, 50: 926

32 Omote M, Eto Y, Tarui A, Sato K, Ando A. Tetrahedron Asymmetry, 2009, 20: 602

33 Nugent W A, RajanBabu T V, Burk M J. Science, 1993, 259: 479

34 Hawkins J M, Watson T J N. Angew Chem, Int Ed, 2004, 43: 3224

35 Mao J C, Wan B S, Wang R L, Wu F, Lu S W. J Org Chem, 2004, 69: 9123

36 Mao J C, Wan B S, Wu F, Lu S W. Chirality, 2005, 17: 245

37 Mao J C, Wan B S, Wu F, Lu S W. J Mol Catal A, 2005, 237: 126

38 Li H W, Huang Y B, Jin W, Xue F, Wan B S. Tetrahedron Lett, 2008, 49: 1686

39 Cernerud M, Adolfsson H, Moberg C. Tetrahedron Asymmetry, 1997, 8: 2655 